\title{
Evaluation of In-Boundary Stress in 2D BEM for Isotropìc Elasticity. A Comparative Study
}

\author{
F.J. Calzado, V. Mantič ${ }^{1}$, F. París \\ Group of Elasticity and Strength of Materials, School of Engineering, University of Seville, \\ Camino de los Descubrimientos s/n, Seville, E-41092, SPAIN
}

\begin{abstract}
The present work deals with the recovery of the in-boundary stress on smooth boundary parts in Boundary Element Method (BEM) in two dimensions. First, two Boundary Integral Representations (BIRs) of tangential derivative of boundary displacements, whose integral kernels multiplying either tangential derivative of displacements or displacements are smooth and bounded, are presented. Two procedures for an in-boundary stress recovery based on these BIRs are developed and analyzed. The first procedure, which directly uses the results obtained from a BEM analysis, displacements and tractions, requires to perform integrations involved in these BIRs either over the real boundary of the solid or over a smooth approximation of the boundary part where the evaluation point is placed. The second procedure, which can be applied on a non-smooth (e.g. polygonal) approximation of the boundary, requires to use in the first BIR introduced here an integral density which is previously smoothed by a local smoothing procedure. The third recovery procedure considered in this work is directly given by a local smoothing procedure of this kind. Results obtained by these three recovery procedures are compared in a series of numerical tests, the best results being obtained by the first procedure developed in this work.
\end{abstract}

\section{INTRODUCTION}

An accurate evaluation of the complete stress tensor at a boundary of a body is an important issue for engineering practice considering that maxima of different failure criteria are typically achieved on or near this boundary. Usually, a Boundary Integral Equation (BIE) formulation of an elastic problem directly involves boundary displacements and tractions as variables (París and Cañas, 1997), but not the so-called in-boundary stress.

The present work is dedicated to the evaluation of the in-boundary stress at boundary points placed at smooth boundary parts and where additionally the stress tensor is continuous. Corners and points with a sudden change of boundary conditions are consequently excluded from the scope of the present work.

Two new Boundary Integral Representations (BIRs) of the tangential derivative of boundary displacements for isotropic elastic solids deduced by Mantič et al. (2002), applying a novel idea introduced recently by $\mathrm{Wu}(2000)$ in the framework of anisotropic elasticity, are briefly presented in Section 2. These BIRs are in some sense equivalent each other, as the second one is obtained by integration by parts of the first one. An advantage of the direct application of these BIRs, denoted

\footnotetext{
${ }^{1}$ For questions please email: mantic@esi.us.es
} 
here as LS approach (because of an application of the Barnett and Lothe tensors $\mathbf{L}$ and $\mathbf{S}$ in their deduction), is that the integrals involving the tangential derivative of boundary displacements (or directly the boundary displacements) are non-singular on smooth boundary parts. This non-singular character avoids the necessity to fulfill very demanding continuity requirements on the boundary element approximation of tangential derivative of displacements (or displacements) that hold for the usually used strongly singular (or hypersingular) BIRs (Krishnasamy et al., 1992; Guiggiani et al., 1992; Gray and Manne, 1993; Graciani et al., 2000). When these continuity requirements are not fulfilled at element junctions, a slow convergence of the in-boundary stress can be observed. Avila et al. (1997) observed such a slow convergence for an $h$-refinement evaluating the inboundary stress at the center of continuous linear elements by the hypersingular BIR.

Section 3 presents a local smoothing technique for evaluation of a continuous piecewise linear approximation of displacement gradient, denoted here as DSC approach, using displacements and tractions obtained from a BEM analysis. This technique is a generalization of an analogous smoothing technique applied with very encouraging results to BEM for potential theory by Mantič et al. (1999) and Graciani et al. (2000).

The continuous approximation of displacement gradient obtained by DSC can be applied as an integral density either in the conventional strongly singular BIR of displacements gradient or in the first 'non-singular' BIR of tangential derivative of boundary displacements presented in this work, in a similar way as used in Mantič et al. (1999) and Graciani et al. (2000). In this paper, this second option is used and is denoted as SSC approach. An advantage of this approach is that it can be applied in a coherent way at corners of a polygonal approximation of the boundary, i.e. at boundary element junctions on originally curved boundary parts.

Results of a comparative numerical study are presented in Section 5. In-boundary stress is evaluated at nodes in the post-processing phase using the following three approaches: LS, DSC, and SSC. The numerical comparative study performed has shown a superior accuracy of the novel LS approach in comparison with the other two approaches. Nevertheless, in several cases, the accuracy obtained by DSC has been very similar to that of LS approach. This fact is very important if the simplicity of implementation of DSC approach in a BEM code and its efficiency, in terms of computational costs, are taken into account.

\section{NOVEL BIRs FOR ISOTROPIC ELASTICITY}

Consider an isotropic elastic body, defined by a plane section domain $\Omega \subset R^{2}$ with a bounded piecewise smooth Lipschitz boundary $\Gamma$, subjected to a plane strain state. Let $G$ and $v$ respectively denote the shear modulus and Poisson ratio. Let $\Gamma_{S} \subset \Gamma$ denote the smooth part of $\Gamma$, for simplicity, $C^{\infty}$ will be assumed.

Let $\mathbf{s}(\mathbf{x})$ and $\mathbf{n}(\mathbf{x})\left(\mathbf{x} \in R^{2}\right)$ be unit vectors perpendicular each other and related by $n_{i}=\varepsilon_{i j} s_{j}$. When $\mathbf{x} \in \Gamma_{S}, \mathbf{s}(\mathbf{x})$ and $\mathbf{n}(\mathbf{x})$ are considered to be the unit tangential and outward normal vectors to the boundary $\Gamma$.

$\mathrm{Wu}$ et al. (1992) introduced a pair of strongly singular BIEs, for tangential derivatives of displacements $\partial_{s} \mathbf{u}$ and tractions $\mathbf{t}$ (associated to $\mathbf{s}(\mathbf{x})$ and $\mathbf{n}(\mathbf{x})$ respectively), which can be written for any $\mathbf{x} \in R^{2}$ except for boundary corners as follows: 


$$
\begin{aligned}
& C(\mathbf{x}) \partial_{s} \mathbf{u}(\mathbf{x})=\text { p.v. } \int_{\Gamma}\left(\frac{\partial \mathbf{U}}{\partial s_{x}}(\mathbf{x}, \mathbf{y}) \mathbf{t}(\mathbf{y})-\frac{\partial \mathbf{W}}{\partial s_{x}}(\mathbf{x}, \mathbf{y}) \partial_{s} \mathbf{u}(\mathbf{y})\right) \mathrm{d} \Gamma_{y}, \\
& -C(\mathbf{x}) \mathbf{t}(\mathbf{x})=\text { p.v. } \int_{\Gamma}\left(\frac{\partial \mathbf{W}^{T}}{\partial s_{x}}(\mathbf{x}, \mathbf{y}) \mathbf{t}(\mathbf{y})-\frac{\partial \mathbf{V}}{\partial s_{x}}(\mathbf{x}, \mathbf{y}) \partial_{s} \mathbf{u}(\mathbf{y})\right) \mathrm{d} \Gamma_{y},
\end{aligned}
$$

$\mathbf{U}, \mathbf{W}$ and $\mathbf{V}$ being imaginary parts of the following $2 \times 2$ complex matrix functions representing fundamental singular elastic solutions:

$$
\begin{aligned}
& \tilde{\mathbf{U}}(\mathbf{x}, \mathbf{y})=\mathbf{U}^{*}+i \mathbf{U}=-\frac{1}{8 \pi G(1-v)}\left[i(3-4 v) \log z \mathbf{I}+i \frac{z-\bar{z}}{2 z}\left(\begin{array}{cc}
1 & i \\
i & -1
\end{array}\right)\right], \\
& \tilde{\mathbf{V}}(\mathbf{x}, \mathbf{y})=\mathbf{V}^{*}+i \mathbf{V}=\frac{G}{2 \pi(1-v)}\left[i \log z \mathbf{I}+i \frac{z-\bar{z}}{2 z}\left(\begin{array}{cc}
1 & i \\
i & -1
\end{array}\right)\right], \\
& \tilde{\mathbf{W}}(\mathbf{x}, \mathbf{y})=\mathbf{W}^{*}+i \mathbf{W}=\frac{1}{4 \pi(1-v)}\left[\log z\left(\begin{array}{cc}
2(1-v) & i(1-2 v) \\
-i(1-2 v) & 2(1-v)
\end{array}\right)+\frac{z-\bar{z}}{2 z}\left(\begin{array}{cc}
1 & i \\
i & -1
\end{array}\right)\right],
\end{aligned}
$$

where $z=\left(x_{1}-y_{1}\right)+i\left(x_{2}-y_{2}\right), i$ is the imaginary unit, $\mathbf{I}$ is the $2 \times 2$ identity matrix. The free term coefficient in (1) fulfills, $C(\mathbf{x})=1$ for $\mathbf{x} \in \Omega, C(\mathbf{x})=1 / 2$ for $\mathbf{x} \in \Gamma_{S}$, and $C(\mathbf{x})=0$ for $\mathbf{x} \notin \Omega \cup \Gamma$. In (1) it is supposed that displacement derivatives are locally Hölder continuous at $\mathbf{x} \in \Omega \cup \Gamma, \mathbf{u} \in C^{l, \alpha}(\mathbf{x})(0<\alpha \leq l)$. The Cauchy principal value integral in (1) for $\mathbf{x} \in \Gamma_{S}$ is denoted by p.v.

Let $\mathbf{L}$ and $\mathbf{S}$ denote $2 \times 2$ Barnett-Lothe tensors (Ting, 1996) defined as:

$$
L_{i j}=\frac{G}{1-v} \delta_{i j}, \quad S_{i j}=-\frac{1-2 v}{2(1-v)} \varepsilon_{i j},
$$

where $\delta_{i j}$ is the Kronecker delta and $\varepsilon_{i j}$ is the permutation symbol $\left(\varepsilon_{12}=-\varepsilon_{21}=1, \varepsilon_{11}=\varepsilon_{22}=0\right)$.

Then, applying a procedure introduced by $\mathrm{Wu}$ (2000) for anisotropic materials, and developed by Mantič et al. (2002) for isotropic materials, the following novel BIE is obtained when BIEs (1) are added multiplying from the left the first one by $\mathbf{L}$ and the second one by $-\mathbf{S}^{T}$ :

$$
C(\mathbf{x}) \mathbf{L} \partial_{s} \mathbf{u}(\mathbf{x})+C(\mathbf{x}) \mathbf{S}^{T} \mathbf{t}(\mathbf{x})=\int_{\Gamma} \frac{\partial \mathbf{V}^{*}}{\partial s_{x}}(\mathbf{x}, \mathbf{y}) \partial_{s} \mathbf{u}(\mathbf{y}) \mathrm{d} \Gamma_{y}-\text { p.v. } \int_{\Gamma} \frac{\partial \mathbf{W}^{* T}}{\partial s_{x}}(\mathbf{x}, \mathbf{y}) \mathbf{t}(\mathbf{y}) \mathrm{d} \Gamma_{y} .
$$

Explicit expressions of the above integral kernels in a real variable formulation can be written as:

$$
\begin{aligned}
& \frac{\partial V_{i j}^{*}}{\partial s_{x}}(\mathbf{x}, \mathbf{y})=-\frac{G}{\pi(1-v) r} r_{, i} r_{, j} r_{, m} n_{m}(x), \\
& \frac{\partial W_{i j}^{*}}{\partial s_{x}}(\mathbf{x}, \mathbf{y})=\frac{1}{4 \pi(1-v) r}\left(-2(1-v) r_{, l} \delta_{i j} \varepsilon_{l m}-(1-2 v) r_{, m} \varepsilon_{i j}+r_{, j} \varepsilon_{i m}+r_{, i} \varepsilon_{j m}-2 r_{, i} r_{, j} r_{l} \varepsilon_{l m}\right) n_{m}(x),
\end{aligned}
$$

where $\mathbf{r}=\mathbf{x}-\mathbf{y}, r=|\mathbf{r}|$ and $r_{, i}=r_{i} / r$. Taking $\mathbf{x} \in \Gamma_{S}$, it can be shown from (5) that the integral kernel $\partial \mathbf{V}^{*}(\mathbf{x}, \mathbf{y}) / \partial s_{x}$ is a smooth function of $\mathbf{y} \in \Gamma_{S}$ and a bounded function for all $\mathbf{y} \in \Gamma$, which implies that the first integral of the right-hand side in (4) is regular.

Considering $\mathbf{x} \in \Gamma_{S}$, the following BIR of $\partial_{s} \mathbf{u}$ can be obtained from (4):

$$
\frac{1}{2} \mathbf{L} \partial_{s} \mathbf{u}(\mathbf{x})=-\frac{1}{2} \mathbf{S}^{T} \mathbf{t}(\mathbf{x})-\text { p.v. } \int_{\Gamma} \frac{\partial \mathbf{W}^{* T}}{\partial s_{x}}(\mathbf{x}, \mathbf{y}) \mathbf{t}(\mathbf{y}) \mathrm{d} \Gamma_{y}+\int_{\Gamma} \frac{\partial \mathbf{V}^{*}}{\partial s_{x}}(\mathbf{x}, \mathbf{y}) \partial_{s} \mathbf{u}(\mathbf{y}) \mathrm{d} \Gamma_{y}
$$


Consider that approximations of $\mathbf{u}$ and $\mathbf{t}$ obtained directly from a BEM analysis are applied in the right-hand side of (6). Then, an important advantage of BIR (6) is the fact that errors originated by a differentiation of an approximation of $\mathbf{u}$ in the last integral of (6) are smoothened by the smooth and bounded character of the integral kernel $\partial \mathbf{V}^{*}(\mathbf{x}, \mathbf{y}) / \partial s_{x}$. In fact, an approximation of $\partial_{s} \mathbf{u}$ in the last integral of (6) does not have to fulfil any continuity requirements along $\Gamma$.

Taking into account the fact that the usual result of a BEM analysis are displacements $\mathbf{u}$ instead of their tangential derivative $\partial_{s} \mathbf{u}$, the following BIR of $\partial_{s} \mathbf{u}$, obtained by an integration by parts of the second integral in (6) for $\mathbf{x} \in \Gamma_{S}$ (Mantič et al., 2002), could be considered even more suitable for applications:

$$
\frac{1}{2} \mathbf{L} \partial_{s} \mathbf{u}(\mathbf{x})=-\frac{1}{2} \mathbf{S}^{T} \mathbf{t}(\mathbf{x})-\text { p.v. } \int_{\Gamma} \frac{\partial \mathbf{W}^{* T}}{\partial s_{x}}(\mathbf{x}, \mathbf{y}) \mathbf{t}(\mathbf{y}) \mathrm{d} \Gamma_{y}-\int_{\Gamma} \frac{\partial^{2} \mathbf{V}^{*}}{\partial s_{y} \partial s_{x}}(\mathbf{x}, \mathbf{y}) \mathbf{u}(\mathbf{y}) \mathrm{d} \Gamma_{y} .
$$

An explicit expression of $\partial^{2} \mathbf{V}^{*}(\mathbf{x}, \mathbf{y}) / \partial s_{y} \partial s_{x}$ can be written as:

$$
\frac{\partial^{2} V_{i j}^{*}(\mathbf{x}, \mathbf{y})}{\partial s_{y} \partial s_{x}}=-\frac{G}{\pi(1-v) r^{2}}\left\{\delta_{i k} r_{, j} r_{, m}+\delta_{j k} r_{, i} r_{, m}+\delta_{m k} r_{, i} r_{, j}-4 r_{, i} r_{, j} r_{k} r_{, m}\right\} \varepsilon_{k l} n_{l}(\mathbf{y}) n_{m}(\mathbf{x}) .
$$

It has to be stressed that for a fixed $\mathbf{x} \in \Gamma_{S}$ this kernel has been obtained as a tangential derivative of a smooth function of $\mathbf{y} \in \Gamma_{S}$. Therefore, the integral kernel $\partial^{2} \mathbf{V}^{*}(\mathbf{x}, \mathbf{y}) / \partial s_{y} \partial s_{x}$ is hypersingular only apparently, being in fact a smooth function for $\mathbf{y} \in \Gamma_{S}$ and bounded for all $\mathbf{y} \in \Gamma$, considering a fixed $\mathbf{x} \in \Gamma_{S}$. Consequently, a BEM approximation of $\mathbf{u}$ applied on the righthand side of (7) is not required to be smooth at boundary element junctions (it could be even discontinuous) in order to obtain a continuous approximation of $\partial_{s} \mathbf{u}$ on the left-hand side in (7).

For $\mathbf{x} \in \Gamma_{S}$, in-boundary stress $\sigma_{s s}(\mathbf{x})$ can be evaluated applying Hooke's law in terms of the normal stress on boundary $t_{n}(\mathbf{x})=\sigma_{n n}(\mathbf{x})$, obtained as a direct result of a BEM analysis, and inboundary strain $e_{s s}(\mathbf{x})=\partial_{s} u(\mathbf{x})$, obtained applying either BIR (6) or (7), as follows:

$$
\sigma_{s s}=\frac{v}{1-v} t_{n}+\frac{E}{1-v^{2}} \partial_{s} u_{s} \text {. }
$$

It can be expected that the accuracy of $\sigma_{s s}$ evaluated in this way will be similar to that of direct results of a BEM analysis: displacements and tractions. The above-described procedure will be referred to as LS approach.

Finally it has to be pointed out that in a BEM application of LS approach, integrations in (6) or (7) could also be performed over a smooth approximation of a curved $\Gamma_{S}$ (e.g. by splines) instead of over the real boundary.

\section{LOCAL SMOOTHING PROCEDURE}

A simple local smoothing procedure for evaluation of displacement gradient $u_{i, j}(\mathbf{x})$ using continuous linear boundary elements can be developed by a generalization of a procedure of this kind for potential problems introduced by Mantič et al. (1999) and Graciani et al. (2000).

Consider a smooth segment of $\Gamma$ approximated by two linear elements $\Gamma_{e}, e=1,2$, defined by three consecutive nodes $\mathbf{x}^{(l)}, \mathbf{x}$, and $\mathbf{x}^{(2)} \in \Gamma$. Let $\mathbf{n}^{(e)}$ and $\mathbf{s}^{(e)}$ respectively be the unit outward 
normal and tangential vectors to $\Gamma^{(e)}$. Let $h_{e}=\left|\mathbf{x}^{(e)}-\mathbf{x}\right|$ and $h_{12}=\left|\mathbf{x}^{(l)}-\mathbf{x}^{(2)}\right|$. Let $\mathbf{n}_{h}(\mathbf{x})$ and $\mathbf{s}_{h}(\mathbf{x})$ denote some approximations (or exact values if available) of the unit outward normal and tangential vectors to $\Gamma$ at $\mathbf{x}$. They can be defined, for example, as follows:

$$
\mathbf{n}_{h}(\mathbf{x})=\frac{h_{2}}{h_{12}} \mathbf{n}^{(l)}+\frac{h_{1}}{h_{12}} \mathbf{n}^{(2)}, \quad \mathbf{s}_{h}(\mathbf{x})=\frac{h_{2}}{h_{12}} \mathbf{s}^{(1)}+\frac{h_{1}}{h_{12}} \mathbf{s}^{(2)} .
$$

Let $\mathbf{t}_{h}(\mathbf{x})$ and $\mathbf{u}_{h}(\mathbf{x})$ respectively denote nodal values of tractions and displacements obtained directly from a BEM analysis. Then the following approximation of the value of $u_{i, j}(\mathbf{x})$ can be obtained using Hooke's law (Wu et al., 1992):

$$
\left(u_{i, j}\right)_{h}(\mathbf{x})=A_{i j k}(\mathbf{x}) t_{h k}(\mathbf{x})+B_{i j k}(\mathbf{x})\left(\partial_{s} u_{k}\right)_{h}(\mathbf{x}),
$$

where

$$
\begin{aligned}
& A_{i j k}(\mathbf{x})=\frac{1}{G}\left(\delta_{i k}-\frac{1}{2(1-v)} n_{h i}(\mathbf{x}) n_{h k}(\mathbf{x})\right) n_{h j}(\mathbf{x}), \\
& B_{i j k}(\mathbf{x})=-\frac{v}{1-v} \varepsilon_{i k} n_{h j}(\mathbf{x})-\frac{1}{1-v} s_{h i}(\mathbf{x}) n_{h k}(\mathbf{x}) n_{h j}(\mathbf{x})+\delta_{i k} s_{h j}(\mathbf{x}),
\end{aligned}
$$

and $\left(\partial_{s} \mathbf{u}\right)_{h}(\mathbf{x})$ is an approximation of the tangential derivative of displacements. It can be defined, for example, as:

$$
\left(\partial_{s} \mathbf{u}\right)_{h}(\mathbf{x})=\frac{h_{2}}{h_{12}} \frac{\partial \mathbf{u}^{(1)}}{\partial s^{(1)}}+\frac{h_{1}}{h_{12}} \frac{\partial \mathbf{u}^{(2)}}{\partial s^{(2)}}, \quad \frac{\partial \mathbf{u}^{(1)}}{\partial s^{(1)}}=\frac{\mathbf{u}_{h}(\mathbf{x})-\mathbf{u}_{h}\left(\mathbf{x}^{(1)}\right)}{h_{1}} \quad \text { and } \quad \frac{\partial \mathbf{u}^{(2)}}{\partial s^{(2)}}=\frac{\mathbf{u}_{h}\left(\mathbf{x}^{(2)}\right)-\mathbf{u}_{h}(\mathbf{x})}{h_{2}}
$$

When $\mathbf{x}$ is placed at a corner of $\Gamma, u_{i, j}(\mathbf{x})$ can be approximated by

$$
\left(u_{i, j}\right)_{h}(\mathbf{x})=\left(e_{i j}\right)_{h}(\mathbf{x})+\omega_{h}(\mathbf{x}) \varepsilon_{i j},
$$

where first, an approximation of the strain tensor $\left(e_{i j}\right)_{h}(\mathbf{x})$ is obtained using Hooke's law from an approximation of the stress tensor $\left(\sigma_{i j}\right)_{h}(\mathbf{x})$, solution (by the least square method) of the following linear system: $\left(\sigma_{i j}\right)_{h}(\mathbf{x}) n_{j}^{(e)}=t_{h i}^{(e)}(\mathbf{x}) \quad(i, e=1,2)$, corresponding to Cauchy lemma applied at $\Gamma^{(e)}$. Second, an approximation of the infinitesimal rotation $\omega_{h}(\mathbf{x})$ is obtained by a solution (by the least square method) of the following linear system: $\partial u_{i}^{(e)} / \partial s^{(e)}=\left(e_{i j}\right){ }_{h}(\mathbf{x}) s_{j}^{(e)}+\omega_{h}(\mathbf{x}) \varepsilon_{i j} s_{j}^{(e)}(i, e=1,2)$.

Let $\Gamma_{h}$ denote an approximation of the real boundary $\Gamma$ by linear boundary elements, $h$ being a characteristic element size in the mesh. Then, a continuous and piecewise linear approximation of displacement gradient, denoted hereinafter as $\left(u_{i, j}\right)_{h}$, over $\Gamma_{h}$ (which obviously defines a continuous and piecewise linear approximation $\left(\sigma_{i j}\right)_{h}$ of stress tensor on $\left.\Gamma_{h}\right)$ is defined by its nodal values given by means of (11) or (14). This procedure is referred to as DSC approach.

\section{APPLICATION OF SMOOTHENED DENSITY IN A BIR}

Consider a fixed point $\mathbf{x} \in \Gamma$ and a fixed unit vector $\mathbf{s}(\mathbf{x})$. Then using a standard asymptotic analysis (see Guiggiani et al. 1992, Mantič and París, 1995), which starts considering BIE (4) for the domain $\Omega \backslash B_{\varepsilon}(\mathbf{x})$ with an excluded circular vanishing zone $B_{\varepsilon}(\mathbf{x})=\left\{y \in R^{2},|\mathbf{y}-\mathbf{x}|<\varepsilon\right\}$, the following general boundary form of BIE (4) can be obtained (Calzado, 2001): 


$$
\mathbf{C}^{\partial_{s} u}(\mathbf{x}) \partial_{s} \mathbf{u}(\mathbf{x})+\mathbf{C}^{t}(\mathbf{x}) \mathbf{t}(\mathbf{x})=\text { p.v. } \int_{\Gamma}\left(\frac{\partial \mathbf{V}^{*}}{\partial s_{x}}(\mathbf{x}, \mathbf{y}) \partial_{s} \mathbf{u}(\mathbf{y})-\frac{\partial \mathbf{W}^{* T}}{\partial s_{x}}(\mathbf{x}, \mathbf{y}) \mathbf{t}(\mathbf{y}) \mathrm{d} \Gamma_{y}\right) .
$$

Somewhat complicated explicit expressions, in a real variable formulation, of the coefficient tensors $\mathbf{C}^{\partial_{s} u}(\mathbf{x})$ and $\mathbf{C}^{t}(\mathbf{x})$ of the free terms in (15) have been deduced by Calzado (2001) and are omitted here for the sake of brevity. Note that in the particular cases when either $\mathbf{x}$ is at a corner or $\mathbf{s}(\mathbf{x})$ does not coincide with the tangential vector to $\Gamma$ at $\mathbf{x}$, the integral kernel $\partial \mathbf{V}^{*}(\mathbf{x}, \mathbf{y}) / \partial s_{x}$ in (15) is actually strongly singular.

Consider BIE (15) rearranged into a form similar to BIR (6). An advantage of the BIR of $\partial_{s} \mathbf{u}$ obtained in this way is that it can be applied directly on $\Gamma_{h}$, in the same way as was done in the original SSC procedure for potential gradient representation in Mantič et al (1999) and Graciani et al. (2000). In this procedure, vector $\mathbf{s}(\mathbf{x})$ used in BIE (15) is defined for a point $\mathbf{x} \in \Gamma_{h}$, which corresponds to a point $\mathbf{x}^{\prime} \in \Gamma_{S}$, by an approximation of the actual tangent vector to $\Gamma$ at $\mathbf{x}^{\prime}$, see e.g. (10). Note that points $\mathbf{x}$ and $\mathbf{x}^{\prime}$ coincide at nodes of $\Gamma_{h}$ and at straight parts of $\Gamma$. Then, continuous and piecewise linear DSC approximations $\left(u_{i, j}\right)_{h}$, and $\left(\sigma_{i j}\right)_{h}$ on $\Gamma_{h}$ (see Section 3) are used to define the integral densities, $\partial_{s} \mathbf{u}(\mathbf{y})$ and $\mathbf{t}(\mathbf{y})$, on the right-hand side of (15), by taking the actual tangent and outward normal vectors to each boundary element of $\Gamma_{h}$. In this way the continuity requirements in order to obtain a reliable BIR of $\partial_{s} \mathbf{u}(\mathbf{x})$ from (15) are fulfilled and neither spurious logarithmic singularities nor scale dependency at element junctions appear. The above-described procedure will be also referred to as SSC approach in the framework of elasticity theory.

\section{NUMERICAL STUDY}

A series of three examples is presented to illustrate the performance of LS, DSC and SSC approaches in recovering in-boundary stress $\sigma_{s s}$ at nodes. Continuous linear boundary elements and collocations at nodes of the boundary discretization of the Somigliana displacement identity with analytical integrations have been applied in the BEM analysis (París and Cañas, 1997). All boundary conditions are given by tractions, removing of rigid body motions being performed by a procedure developed in Blázquez et al. (1996).

First, two examples motivated by bending of beams problems with exclusively straight boundary parts, beam in pure bending and simply supported beam under a uniform load, are solved. Dimensions of domains are $L \times H, L=2 \mathrm{~cm}, H=1 \mathrm{~cm}$. Then, an infinite plate with a circular hole of radius $R=1 \mathrm{~cm}$ subjected to a uniaxial tension at infinity is solved. Uniform meshes obtained by an $h$-refinement, starting from a basic mesh (with 12 and 8 elements respectively in case of beams and plate with a hole) and partitioning successively each element to three equally large smaller elements, are applied to each problem in order to study solution convergence. Note that in the LS approach the integrals are evaluated over the real boundary (i.e., in case of the circular hole over the circle). Results for several meshes and all approaches studied are presented for each problem. Convergences of characteristic (e.g. maximum) values of in-boundary stresses evaluated by the three approaches studied are compared in log-log scales for each problem solved.

Material properties in all examples have been defined as Young modulus $E=200 \mathrm{MPa}$ and Posisson ratio $v=0.25$. Characteristic load value in all examples is $p=1 \mathrm{MPa}$. Analytic solutions of the problems analyzed are given in Timoshenko and Goodier (1970). 


\subsection{Results for beams in bending}

Results obtained in the recovery of $\sigma_{s s}$ are shown in Figs. 1 and 2, the basic mesh and a simple sketch of the problems solved being attached.

As can be seen in Fig.1 the best results in pure bending problem have been obtained by LS and the worst by SSC approach. Note that the difference between these two approaches, both based on the same BIR (6), are the integral densities used. In this particular case of straight boundaries the difference consists only in the approximations of $\partial_{s} \mathbf{u}(\mathbf{y})$ applied on the right-hand side of (6). In the LS approach the approximation of $\partial_{s} \mathbf{u}(\mathbf{y})$ applied is piecewise constant and thus discontinuous, whereas in SSC approach is piecewise linear and continuous (with obvious exception at corner points). As has been mentioned in Graciani et al. (2000) an explanation of the worse results by SSC can be related to the fact that the condition of vanishing of the integral of $\partial_{s} \mathbf{u}$ over the whole boundary is not exactly fulfilled by $\partial_{s} \mathbf{u}(\mathbf{y})$ in SSC, whereas it is obviously exactly fulfilled by the approximation of $\partial_{s} \mathbf{u}(\mathbf{y})$ in LS.

In Fig. $1 \mathrm{~b}$ an increase of errors near corners can be observed, the lowest errors being provided by LS approach. It appears that this increase is related with an increase of the error in displacements obtained directly in BEM analysis (Calzado, 2001). A stable quadratic convergence $O\left(h^{2}\right)$ (or equivalently $O\left(N^{-2}\right)$, N being the number of nodes in each mesh) of errors obtained by all recovery procedures has been observed for all nodes (except for corners) of the basic mesh. Fig. $1 \mathrm{c}$ shows a typical convergence behaviour for the centre of the bottom side.

When a more complicated problem, simply supported beam under a uniform load, whose solution in displacement is a polynomial of fourth order, is considered, the relative performance of the three approaches studied is different in comparison with the previous problem. Note that a local maximum in $\sigma_{s s}$ is situated at the centre of the horizontal sides. Although, as can be seen in Fig. 2, LS is again the best approach, the difference between DSC and SSC is not so clear, SSC being better on horizontal sides and DSC on vertical sides. Typical increase of error near corners is also present here, although another increase of error takes place at local stress maximum as well, as could be expected. A stable $O\left(h^{2}\right)$ convergence of all approaches has been obtained for all nodes of the basic mesh, and it is shown for the local stress maximum values in Fig. 2c.

\subsection{Results for plate with a circular hole in tension}

The problem of a plate with a circular hole in a uniaxial tension has been solved in BEM analysis as an external problem using a standard superposition procedure (París and Cañas, 1997). As can be seen in Fig. 3, excellent results in the evaluation of hoop stress have been obtained by the LS approach. Recall that in this approach integrations in BIR (6) have been performed over the real circular boundary with a piecewise linear approximation of $\mathbf{t}(\mathbf{y})$ and a piecewise constant approximation of $\partial_{s} \mathbf{u}(\mathbf{y})$. On the contrary, in SSC they have been performed over a polygonal boundary element approximation of circle. An expected increase of error has been obtained at both local stress maxima, higher errors being obtained at stress concentration point. $O\left(h^{2}\right)$ convergence has been observed at all nodes of the basic mesh, Fig. $3 \mathrm{c}$ showing this convergence at local stress maxima. 


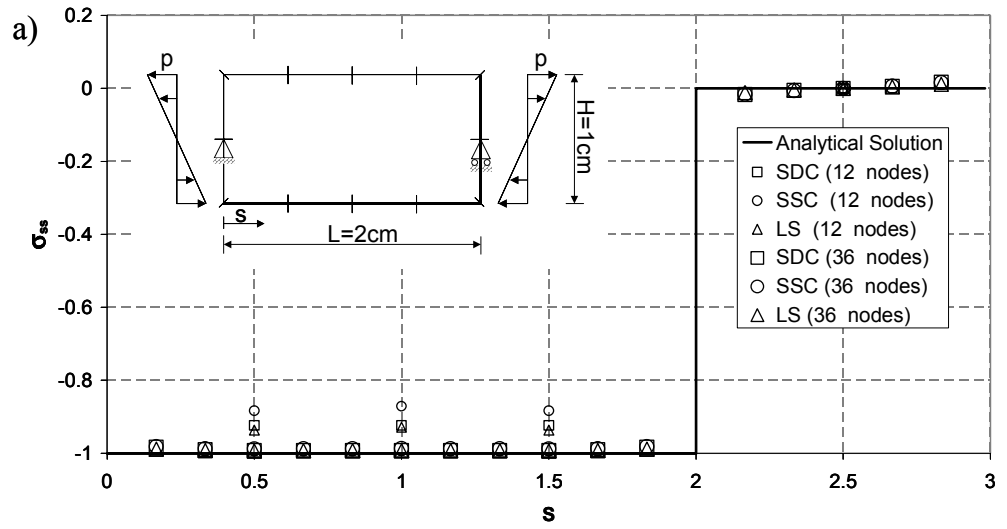

b)
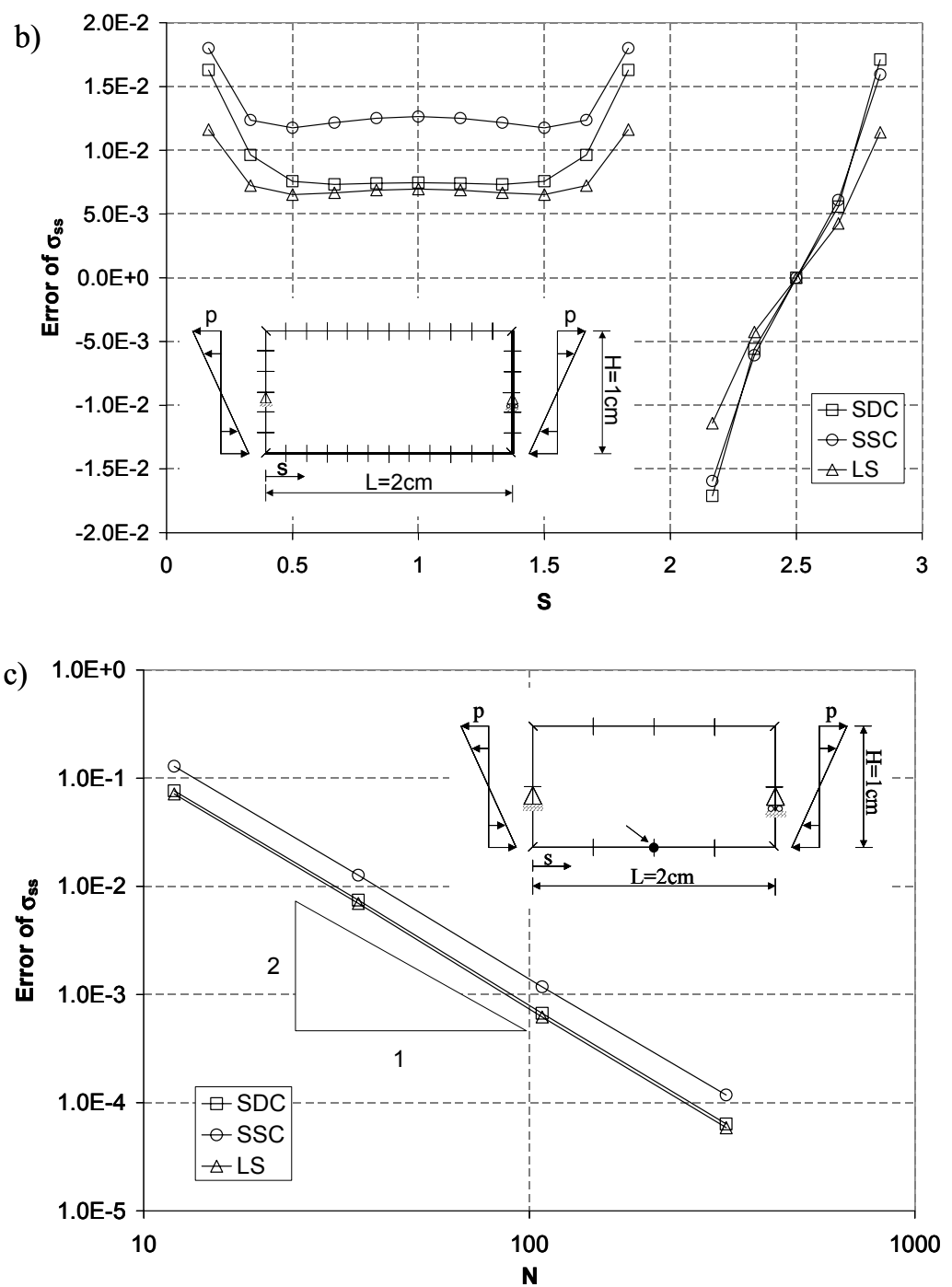

Figure 1: Pure bending problem. Linear boundary elements. a) In-boundary stress evaluated at nodes. Meshes of 12 and 36 elements. b) Absolute errors of the in-boundary stress evaluated at nodes. Mesh of 36 elements. c) Convergence of the absolute error of the in-boundary stress evaluated at the center of the bottom side. 
a)
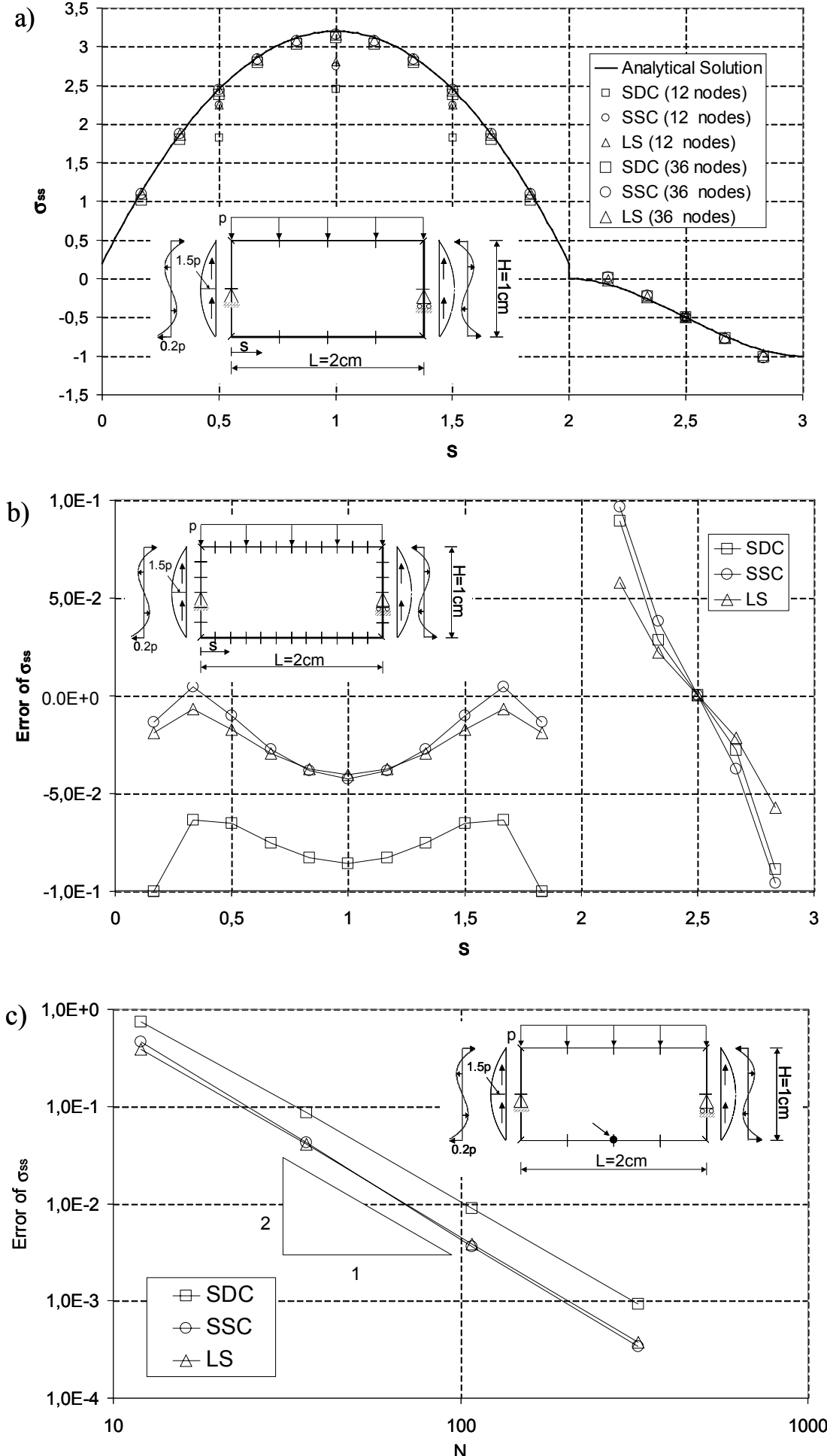

Figure 2: Simply supported beam under uniform load. Linear boundary elements. a) In-boundary stress evaluated at nodes. Meshes of 12 and 36 elements. b) Absolute errors of the in-boundary stress evaluated at nodes. Mesh of 36 elements. c) Convergence of the absolute error of the inboundary stress evaluated at the center of the bottom side. 

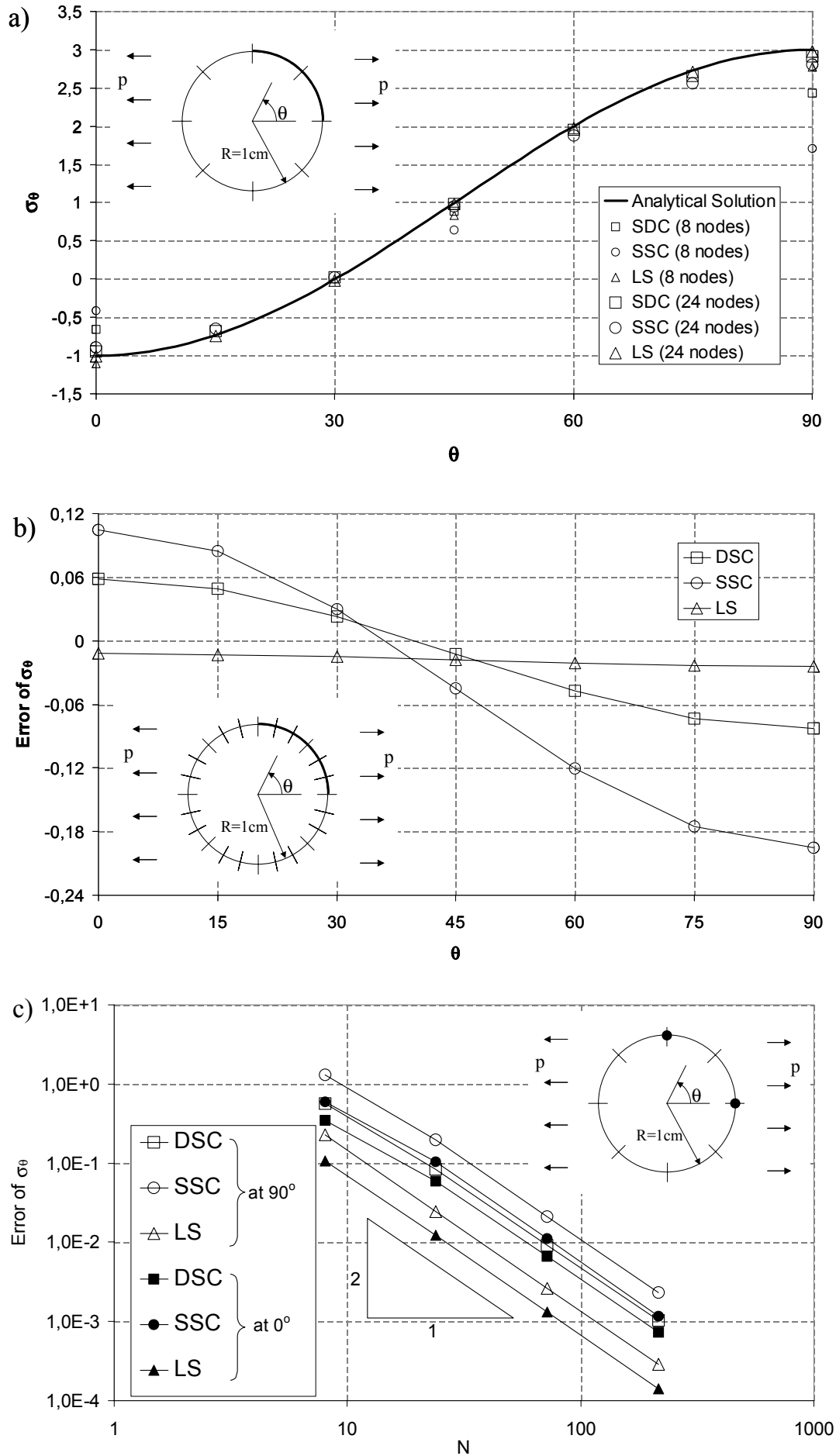

Figure 3: Plate with a circular hole in tension. Linear boundary elements. a) Hoop stress evaluated at nodes. Meshes of 8 and 24 elements. b) Absolute errors in the hoop stress evaluated at nodes. Mesh of 24 elements. c) Convergence of the absolute error in the hoop stress evaluated at $\theta=0^{\circ}$, $90^{\circ}$. 


\section{CONCLUSIONS}

A novel approach, denoted as LS in this paper, for recovery of the in-boundary stress $\sigma_{s s}$ in BEM has been developed applying one of two new BIRs of $\partial_{s} \mathbf{u}$ introduced in the present work. These BIRs are equivalent to one BIE from a system of BIEs of the second kind recently deduced by Wu (2000) and Mantič et al. (2002). A basic advantage of this LS approach is the fact that, when integrations in these BIRs are performed over the smooth, either real or approximated, parts of the boundary, no demanding continuity requirements have to be fulfilled by an approximation of tangential derivative of displacements (or displacements) used as the integral density. This is due to the smooth and bounded character of the integral kernels which multiply these tangential derivative of displacements (or displacements) in the new BIRs.

Two other approaches for recovery of $\sigma_{s s}$, namely DSC and SSC, have been developed generalizing analogous approaches for recovery of potential gradient developed recently by Mantič et al. (1999) and Graciani et al. (2000).

Numerical tests of recovering $\sigma_{s s}$ at nodes of continuous linear boundary element meshes have shown an excellent performance of the LS approach when compared with the DSC and SSC approaches. A stable quadratic convergence of nodal values of $\sigma_{s s}$, the same as that of direct results of a BEM analysis, $\mathbf{u}$ and $\mathbf{t}$, has been obtained by all recovery approaches tested.

\section{ACKNOWLEDGEMENT}

The work was supported by the Spanish Ministry of Education and Culture under grant No. PB981118. The authors thank to E. Graciani and R. Avila for their BEM code used to solve elasticity problems in this work.

\section{References}

Avila, R., Mantič, V. and París, F. (1997). Application of the Boundary Element Method to Elastic Orthotropic Materials in 2D: Numerical Aspects. In Boundary Elements XIX, CMP Sourthampton, pp. 55-64.

Blázquez, A., Mantič, V., París, F., and Cañas, J. (1996). On the Removal of Rigid Body Motions in the Solution of Elastostatic Problems by Direct BEM. Int. J. for Numerical Methods in Engineering, 39: pp. 4021-4038.

Calzado, F. J. (2001). Evaluation of Stress Tensor at the Boundary by BEM in Plane Elasticity (in Spanish), Final Project, School of Engineering, University of Seville.

Graciani, E., Mantič, V. París, F. and Cañas, J. (2000). A Critical Study of Hypersingular and Strongly Singular Boundary Integral Representations of Potential Gradient. Computational Mechanics, 26(6): pp. 542-559.

Gray, L.J. and Manne, L.L.(1993). Hypersingular Integrals at a Corner. Engineering Analysis with Boundary Elements, 11: pp. 327-334. 
Guiggiani, M., Krishnasamy, G., Rizzo, F.J. and Rudolphi, T.J. (1992). A General Algorithm for the Numerical Solution of Hypersingular Boundary Integral Equations. J. Appl. Mech., 59: pp. 604-614.

Krishnasamy, G., Rizzo, F.J. and Rudolphi, T.J. (1992). Continuity Requirements for Density Functions in the Boundary Integral Equation Method. Computational Mechanics, 9: pp. 267284.

Mantič, V., Graciani, E. and París, F. (1999). A Simple Smoothing Scheme in Strongly Singular Boundary Integral Representation of Potential Gradient. Comput. Methods Appl. Mech. Engrg., 178: pp. 267-289.

Mantič, V., Calzado, F.J. and París, F. (2002). Novel Boundary Integral Equations for 2D Isotropic Elasticity. An Application to Evaluation of the In-Boundary Stress. (submitted for publication).

Mantič, V. and París, F. (1995). Existence and Evaluation of the Two Free Terms in the Hypersingular Boundary Integral Equation of Potential Theory. Engineering Analysis with Boundary Elements, 16: pp. 253-260.

París, F. and Cañas, J. (1997). Boundary Element Method, Fundamentals and Applications. Oxford University Press, Oxford.

Timoshenko, S.P, and Goodier, J. N. (1970). Theory of Elasticity. 3rd ed., McGraw Hill, New York.

Ting, T.C.T., (1996). Anisotropic Elasticity, Theory and Applications. Oxford University Press, New York.

Wu, K.-C., Chiu, Y.-T., Hwu, Z.-H. (1992). A New Boundary Integral Equation Formulation for Linear Elastic Solids. J. Appl. Mech., 59: pp. 344-348.

Wu, K.-C. (2000). Non-singular Boundary Integral Equations for Two-Dimensional Anisotropic Elasticity, J. Appl. Mech., 67: pp. 618-621. 